\title{
Smoker Desaturation during General Anaesthesia: A Case Report
}

\author{
Hadi $M A^{a}$, AzrinaMRa ${ }^{a}$ Zamzila $A^{b}$, Ariff $O^{a}$

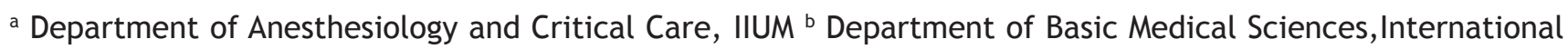 \\ Islamic University Malaysia.
}

\section{ABSTRACT:}

We report a case of sudden hypoxaemia after intubation in a patient who had smoked a few hours prior to a surgical procedure. The cause of his desaturation was not related to bronchial secretions, bronchospasm or obstruction of the upper airways but most likely due to reduced oxygen saturation in the body prior to surgery. We managed to secure the airway and prevent prolonged desaturation by instituting remedial measures. Our conclusion is that cessation of smoking is very important and need to be emphasized in all patients having surgery under general anaesthesia. This applies to emergency cases as well.

KEYWORDS: Smoking; General anaesthesia, Desaturation.

\section{INTRODUCTION:}

Cigarette smoking and its consequences constitute a worldwide epidemic and is attributed to at least $20 \%$ of all deaths in developed countries. ${ }^{1}$ Cigarette smoke contains over 4700 additional chemical compounds other than nicotine. It includes at least 43 carcinogens which collectively generate a very broad range of pathophysiological effects. ${ }^{2}$ Failure to quit smoking before elective or emergency surgery is ill judged which can lead to subsequent risk of intraand postoperative complications. ${ }^{3}$

Smoking up to the time of any surgery increases cardiac and pulmonary complicartions, impairs tissue healing, and is assorciated with more infections and other complications at the surgical site. ${ }^{3,4}$ The relative risk of complications after surgery for smokers compared to nonsmoker has been reported to increase from 1.4-fold to 4.3 -fold.5 These adverse effects compromise the intended procedural outcomes and increase the costs of care. The preoperative period may offer an opportunity for smoking cessation in surgical patients. All available measures must be taken to help patients to stop smoking prior to surgery. It is both responsible and ethical to implement a policy that those unwilling or unable to stop should have low priority for, or be excluded from, certain elective surgical procedures.

Correspondence author:

Dr. Rozilah @ Abdul Hadi Bin Mohamed Department of Anaesthesiology Kulliyyah of Medicine International Islamic University Malaysia Tel: 019-9592256 email:abhadi71@iiu.edu.my

\section{CASE REPORT}

A 44-year-old Indian man was admitted to the casualty with a history of alleged motor vehicle accident. He sustained a deep lacerated wound of $8 \times 2 \mathrm{~cm}$ with exposed bone on the forehead. He denied any history of loss of consciousness, ear, nose or throat bleeding, shortness of breath or abdominal pain. He was able to walk into the casualty department. On examination, he was conscious and alert, and his pupils were reactive $2 \mathrm{~mm}$ bilaterally. His skull X-ray did not revealed any fracture. He was otherwise healthy and was classified as American Society of Anaesthesiology (ASA) class 1. A history of smoking was elicited, and he admitted that he last smoked a few minutes prior to the accident. He was posted for emergency wound toilet and suturing of the lacerated wound under general anaesthesia. His baseline oxygen saturation was $98 \%$. Other baseline parameters were normal. Preoxygenation with $100 \%$ oxygen was administered for 5 minutes to prevent hypoxaemia during the intubation process. He was induced with intravenous fentanyl $75 \mathrm{ug}$, sodium thiopentone $250 \mathrm{mg}$ and suxamethonium $75 \mathrm{mg}$. Intubation was performed with cricoid pressure and intubation was easy by using a endotracheal tube (ETT) size $8.0 \mathrm{~mm}$ with Lehane Cromack grade I. There were no secretions or spasm detected during the process. Endotracheal position was confirmed by auscultation. He was ventilated by using ULCO ventilator with tidal volume setting of $550 \mathrm{ml}$, respiratory rate of $10 / \mathrm{min}$, inspiratory: expiratory ratio of (I:E) $1: 2$ and $02:$ N2O ratio of $1: 2$.

After intubation his oxygen saturation gradually dropped to $94-95 \%$. Auscultations of the lungs were clear and equal on both sides. However, thick secretions was noted in the ETT which was not present during the intubation. Vigorous suctioning and chest physiotherapy were performed. Partial pressure of inspired oxygen (FIO2) was increased to 1.0 since the saturation dropped further to $90-95 \%$. Following this his oxygen saturation gradually picked up to $97-98 \%$ 
with $\mathrm{FIO2}$ of 1.0. The surgery proceeded smoothly with minimal blood loss. He was subsequently reversed and extubated once he was fully awake with good cough reflexes. Prior to extubation, vigorous suctioning and chest physiotherapy was again performed. He was put on facemask oxygen of $6 \mathrm{l} / \mathrm{min}$. However after $10 \mathrm{~min}$ utes, his oxygen saturation suddenly dropped to $54 \%$ and end tidal $\mathrm{CO} 2$ (ETCO2) increased (from $38 \mathrm{mmHg}$ to $67 \mathrm{mmHg}$ ). The patient was restless and complained of difficulty in breathing. Auscultation of the lung revealed generalized transmitted sounds. His oxygen saturation did not improve despite being on high flow mask of $15 \mathrm{~L} / \mathrm{min}$ and continuous nebulizer. A decision for reintubation was made. Continuous aeronebuliser was administered through the ETT and vigorous suctioning and chest physiotherapy was performed. Intravenous hydrocortisone $200 \mathrm{mg}$ was administered. Once the lungs were clear, trial of extubation was performed. Extubation was uneventful and he was observed in the recovery room for 1 hour before being discharged to the general ward.

\section{DISCUSSION}

Postoperative pulmonary complications in smokers are related to the mechanical and chemical properties of the tobacco and smoke. Important respiratory system changes associated with smoking include mucus hypersecretion and impaired tracheobroncial clearance, as well as small airway narrowing with increased closing capacity and tendency to ventilation-perfusion mismatching. Mechanically, smoking impairs mucus transport by causing cilliary damage which lead to increase secretions. Nicotine in cigarette can increase reflex sensitivity of both the upper and lower conducting airways, increased permeability of the respiratory epithelium, and there is also some evidence of loss of surfactant. ${ }^{6}$ Our patient who smoked just a few minutes prior to the accident developed desaturation during the operation and also after the reversal even though the airways were secured. In our case, we have already excluded the possibilities of medical illnesses such as asthma or COAD and there was no family history of similar problems. Although it is known that chronic smokers are prone to have bronchospasm or hypersecretion, the presentation in this patient was unusual. Despite the secured airways, he developed desaturation during the procedure.

Further effects of smoking with implications for the anaesthetist are impaired humoral and cell-mediated immunity and induction of hepatic microsomal enzymes leading to increased metabolism of various drugs. ${ }^{3}$ Cyanide in smoke also inhibit the mitochondrial oxidative metabolism. ${ }^{1}$

Nicotine is a potent vasoconstrictor. It is known to increase arterial carbon monoxide which reduces $\mathrm{CO} 2$ transport. There is also an assault on the cardiovascular system in the form of increased oxygen demand through nicotine activation of the sympatho-adrenergic system, coupled with decreased oxygen supply via raised carboxyhaemoglobin $(\mathrm{COHb})$ levels and raised coronary vascular resistance. ${ }^{2}$

Smokers have lower arterial oxygen tensions pre-operatively. There are studies suggesting that they are more prone than non-smokers to desaturation after induction or intravenous sedation or during recovery from anaesthesia. ${ }^{2}$ Cessation of smoking immediately prior to the operation does not confer an increased risk of pulmonary complications but instead benefit the patients. ${ }^{3,5}$ Smoking cessation $12 \mathrm{~h}$ before surgery significantly reduces carboxyhaemoglobin concentrations, improves oxygen availability, and reverses negative inotropic and arrhythmic effects. ${ }^{3,5} \mathrm{An}$ increase in the heart rate, blood pressure and peripheral vasoconstriction will improve after 12-24 hours of abstinence from smoking. ${ }^{3,5}$

Adherence to published guidelines on pre-operative abstinence would alleviate this type of high-risk morbidity. Cessation of smoking would reduce the acute effects of nicotine and in most cases reduce $\mathrm{COHb}$ levels to those found in non-smokers. ${ }^{3,5}$ Even short periods of abstinence might significantly influence the outcome, in view of the relatively short-elimination half lives of nicotine (30 to $60 \mathrm{~min}$ ) and $\mathrm{COHb}(4$ hours). ${ }^{2,3}$ Many anaesthetists seldom or never make a point of requesting pre-operative abstinence and it is not uncommon for heavy smokers to continue smoking until the time they are taken to the operating theatre. Given that heavy smokers typically have $\mathrm{COHb}$ levels of 5 to $15 \%$, anaesthesia might be induced in a patient whose oxygen saturation is actually $15 \%$ lower than that indicated on the pulse oximeter (oxygen saturation is defined as: oxygen combined with haemoglobin/oxygen capacity $\times 100)^{2,3}$ In fact, available oxygen will still be further decreased, because of $\mathrm{COHb}$-induced left shift in the oxygen dissociation curve. Although our patient during reversal and extubation, did not show any feature of upper airway obstruction due to secretions or any rhonchi because of airway irritation, he still had desaturation despite good oxygen supplement.

Smoking cessation can be encouraged at any time before surgery. The benefits of short-term pre-operative abstinence are essentially confined to the cardiovascular system. ${ }^{2,3}$ Achievement of this minimum goal will require all anaesthetists to take this issue seriously. This should also involve surgeons and nursing staff who are in a position to communicate with the smoking patient preoperatively. Interdisciplinary guidelines similar to those for pre-operative fasting are required. In addition, local policies should be implemented to ensure that day surgery patients are also targeted. It is vital to liaise with surgical colleagues to formulate a joint approach to the problem. A written pre-operative advice sheet for the patient would be a simple way to commence this process. Possibly more effective approaches would be the establishment of and referral to an antismoking clinic. Another strategy could be the funding for a dedicated health educator to target smokers awaiting surgery. 


\section{CONCLUSION}

Growing evidence suggested that smoking in the perioperative period is harmful. Even with a short exposure to smoke prior to general anaesthesia, the potential risk of hypoxaemia is still high. This could occur either during intubation or extubation. Thus preoperative abstinence is beneficial and should be strongly recommended by anesthesiologists.

\section{REFERENCE}

1. Moore S, Mills BB, Moore RD, Miklos JR, Mattox TF. Perisurgical smoking cessation and reduction of postoperative complications. Am J Obstet Gynecol 2005; 192:1718-21

2. Nel MR, Morgan M. Smoking and Anaesthesia Revisited. Anaesthesia 1996; 51:309-11

3. Warner DO. Preoperative Smoking Cessation: The Role of the Primary Care Provider. Mayo Clin Proc 2005; 80:252-8

4. Warner DO. Smoking Behaviour and Perceived Stress in Cigarette Smokers Undergoing Elective Surgery. Surv Anesthesiol 2005; 49:213

5. Warner DO. Tobacco dependence in surgical patients. Curr Opin Anaesthesiol 2007; 20:279- 83 
